\title{
Impact of immunosuppression on incidence, aetiology and outcome of ventilator-associated lower respiratory tract infections
}

Anne-Sophie Moreau ${ }^{1}$, Ignacio Martin-Loeches ${ }^{2}$, Pedro Povoa ${ }^{3,4}$, Jorge Salluh $^{5}$, Alejandro Rodriguez ${ }^{6}$, Arnaud W. Thille ${ }^{7}$, Emilio Diaz Santos ${ }^{8}$, Elisa Vedes ${ }^{9}$, Suzana Margareth Lobo ${ }^{10}$, Bruno Mégarbane ${ }^{11}$, Esperanza Molero Silvero ${ }^{12}$, Luis Coelho $\mathbb{1}^{3,4}$, Laurent Argaud ${ }^{13}$, Rafael Sanchez Iniesta ${ }^{14}$, Julien Labreuche ${ }^{15}$, Anahita Rouzé ${ }^{1}$ and Saad Nseir ${ }^{1,16}$ for the TAVeM Study Group $^{17}$

@ERSpublications

Ventilator-associated lower respiratory tract infections are less common in immunocompromised patients http://ow.ly/p4Ew30ia2fO

Cite this article as: Moreau A-S, Martin-Loeches I, Povoa P, et al. Impact of immunosuppression on incidence, aetiology and outcome of ventilator-associated lower respiratory tract infections. Eur Respir J 2018; 51: 1701656 [https://doi.org/10.1183/13993003.01656-2017].

ABSTRACT The aim of this planned analysis of the prospective multinational TAVeM database was to determine the incidence, aetiology and impact on outcome of ventilator-associated lower respiratory tract infections (VA-LRTI) in immunocompromised patients.

All patients receiving mechanical ventilation for $>48 \mathrm{~h}$ were included. Immunocompromised patients $(\mathrm{n}=663)$ were compared with non-immunocompromised patients $(\mathrm{n}=2297)$.

The incidence of VA-LRTI was significantly lower among immunocompromised than among nonimmunocompromised patients ( $16.6 \%$ versus $24.2 \%$; sub-hazard ratio $0.65,95 \%$ CI $0.53-0.80 ; \mathrm{p}<0.0001$ ). Similar results were found regarding ventilator-associated tracheobronchitis $(7.3 \%$ versus $11.6 \%$; subhazard ratio $0.61,95 \%$ CI $0.45-0.84 ; \mathrm{p}=0.002)$ and ventilator-associated pneumonia $(9.3 \%$ versus $12.7 \%$; sub-hazard ratio $0.72,95 \%$ CI $0.54-0.95 ; \mathrm{p}=0.019)$. Among patients with VA-LRTI, the rates of multidrugresistant bacteria $(72 \%$ versus $59 \% ; \mathrm{p}=0.011)$ and intensive care unit mortality were significantly higher among immunocompromised than among non-immunocompromised patients (54\% versus 30\%; OR 2.68, 95\% CI 1.78-4.02; $\mathrm{p}<0.0001)$. In patients with ventilator-associated pneumonia, mortality rates were higher among immunocompromised than among non-immunocompromised patients (64\% versus 34\%; $\mathrm{p}<0.001)$.

Incidence of VA-LRTI was significantly lower among immunocompromised patients, but it was associated with a significantly higher mortality rate. Multidrug-resistant pathogens were more frequently found in immunocompromised patients with VA-LRTI. 
Affiliations: ${ }^{1}$ Centre de Réanimation, CHU Lille, Lille, France. ${ }^{2}$ Dept of Clinical Medicine, Trinity College, Welcome Trust-HRB Clinical Research Facility, St James Hospital, Dublin, Ireland. ${ }^{3}$ Unidade de Cuidados Intensivos Polivalente, Hospital de São Francisco Xavier, Centro Hospitalar de Lisboa Ocidental, Lisbon, Portugal. ${ }^{4}$ NOVA Medical School, CEDOC, Universidade Nova de Lisboa, Lisbon, Portugal. ${ }^{5}$ Dept of Critical Care, D'Or Institute for Research and Education, Rio De Janeiro, Brazil. ${ }^{6}$ Critical Care Dept, Joan XXIII University Hospital, Tarragona, Spain. ${ }^{7} \mathrm{CHU}$ de Poitiers, Réanimation Médicale, Faculté de Médecine et de Pharmacie de Poitiers, Université de Poitiers, INSERM, CIC-1402, équipe 5 ALIVE, Poitiers, France. ${ }^{8}$ Critical Care Center, Sabadell Hospital, Corporación Sanitaria Universitaria Parc Taulí, Universitat Autonoma de Barcelona, CIBER de Enfermedades Respiratorias (CIBERES), Sabadell, Spain. ${ }^{9}$ Unidade de Cuidados Intensivos do Hospital da Luz, Lisbon, Portugal. ${ }^{10}$ Faculdade de Medicina de São José do Rio Preto, São José do Rio Preto, Brazil. ${ }^{11}$ Dept of Medical and Toxicological Critical Care, Lariboisière Hospital, Paris-Diderot University, INSERM UMRS-1144, Paris, France. ${ }^{12}$ Intensive Medicine Unit, Gómez Ulla Hospital, Madrid, Spain. ${ }^{13}$ Service de Réanimation Médicale, Hôpital Edouard Herriot, Hospices Civils de Lyon, Lyon, France. ${ }^{14}$ General de Albacete, Albacete, Spain. ${ }^{15} \mathrm{CHU}$ Lille, Clinique de Santé Publique, Plateforme d'Aide Méthodologique, Lille, France. ${ }^{16}$ Medical School, Lille University, Lille, France. ${ }^{17}$ For a full list of the TAVeM Study Group members, please refer to the footnote at the end of the article.

Correspondence: Saad Nseir, Centre de Réanimation, Hôpital Salengro, CHRU Lille, 59037 Lille Cedex, France. E-mail: s-nseirlachru-lille.fr

\section{Introduction}

Ventilator-associated lower respiratory tract infections (VA-LRTI) are the most common infectious complication in the intensive care unit (ICU) [1, 2]. They include both ventilator-associated tracheobronchitis (VAT) and ventilator-associated pneumonia (VAP). VAT has been proposed as an intermediate stage between colonisation of the lower respiratory tract and VAP [3, 4]. This infection is associated with higher rates of subsequent VAP, and prolonged duration of mechanical ventilation [5-7]. Recently, our group have shown that VAT is a separate entity, responsible for the increased duration of mechanical ventilation and increased ICU length of stay in a large cohort of medical and surgical patients [2]. VAT was not associated with increased mortality rates, but transition from VAT to VAP was a risk factor for mortality and appropriate antibiotic treatment was protective. Further, several previous studies have demonstrated that VAP is associated with increased morbidity, mortality and cost in critically ill patients [8-11]. However, the mortality attributable to VAP is still a matter for debate $[10,12]$.

Immunocompromised patients have a particularly poor outcome in the ICU, due to a higher risk of infection (especially to opportunistic pathogens), higher severity of illness, immunosuppression itself and poor performance status $[13,14]$. They often receive broad-spectrum antibiotic treatment, thus increasing the risk for developing multidrug-resistant bacteria (MDR) [15]. The main cause of admission to the ICU for these patients is acute respiratory failure [16]. Although their outcomes have substantially improved in recent years, the prognosis remains poor, with hospital mortality rates of up to $60 \%$ in mechanically ventilated patients [17]. However, to our knowledge, no study to date has specifically evaluated VA-LRTI in this population. We hypothesised that immunocompromised patients would develop more VA-LRTI than non-immunocompromised patients, given the context of altered host defences. Therefore, we conducted this study to determine the incidence, aetiology and outcome of these infections in immunocompromised patients, and to determine if these factors are different in patients with no apparent immunosuppression.

\section{Material and methods \\ Patients}

This study is a planned analysis of the large multinational TAVeM database, which prospectively followed patients $>18$ years admitted to 114 ICUs in Europe and Latin America receiving mechanical ventilation for $>48$ h between September 1, 2013 and July 31, 2014 [2].

Participating centres either received ethical approval from their institutions or ethical approval was waived (institutional review board number 2013515). Informed consent was waived because of the observational nature of the study.

Patients considered to be immunocompromised were those with ongoing neoplasia, haematological malignancy, AIDS, an allogeneic stem cell transplant, an organ transplant or taking immunosuppressive drugs [18].

\section{Procedures and definitions}

Patients were prospectively followed for outcome until death or ICU discharge. Demographic data were obtained along with clinical data that included comorbidities, prognostic scores, antibiotic use and diagnostic procedures for VAP and VAT. 
The diagnosis of VA-LRTI was based on the presence of at least two of the following criteria: body temperature $<36.5^{\circ} \mathrm{C}$ or $>38.5^{\circ} \mathrm{C}$, leukocyte count $<4000$ cells $\mu \mathrm{L}^{-1}$ or $>12000$ cells $\mu \mathrm{L}^{-1}$, and purulent endotracheal aspirate. To be included in the final analysis, all episodes of infection had to have a positive microbiological isolation in the endotracheal aspirate of at least $10^{5} \mathrm{CFU} \cdot \mathrm{mL}^{-1}$, or in bronchoalveolar lavage fluid of at least $10^{4} \mathrm{CFU} \cdot \mathrm{mL}^{-1}$.

VAT was defined with the aforementioned criteria with no radiographic signs of new infiltrate; VAP was defined by the presence of new or progressive infiltrate on chest radiography. VAP was deemed to occur subsequently to VAT if it was diagnosed in the $96 \mathrm{~h}$ period after diagnosis of tracheobronchitis and if the same microorganism caused both infections. VAP was considered to be early-onset when it was diagnosed $<5$ days, and late-onset when it was diagnosed $\geqslant 5$ days, after starting mechanical ventilation [19].

Empirical antibiotic therapy was defined as that given before microbiological documentation of infection. Antibiotic treatment was considered appropriate when at least one antibiotic, active in vitro on all organisms causing VA-LRTI, was administrated to treat these infections [20]. Microbiological identification and susceptibility tests were performed using standard methods. MDR was defined as acquired non-susceptibility to at least one agent in three or more antimicrobial categories [21]. More details on methods are available in the TAVeM principal paper [2].

\section{Outcomes}

The primary aim of our study was to determine the incidence of VA-LRTI, including VAT and VAP, comparing immunocompromised patients and non-immunocompromised patients. Our secondary objectives were to determine the aetiology and impact on outcome (length of stay in the ICU and hospital, days with mechanical ventilation, and mortality) of VA-LRTI in immunocompromised patients as compared to non-immunocompromised patients. We also studied the effect of appropriate antibiotic treatment on the progression from VAT to VAP in immunocompromised patients as compared to in non-immunocompromised patients.

\section{Statistical analysis}

We used SPSS software (version 22; SPSS Inc., IBM, New York, NY, USA) for data analysis. The incidence, aetiology and outcome of VA-LRTI were compared between immunocompromised and non-immunocompromised patients. Only first episodes of VAT and VAP were taken into account.

Categorical variables were described as numbers and frequencies (\%), normally distributed continuous variables as mean $\pm \mathrm{SD}$ and skewed continuous variables as medians (interquartile range (IQR)). We used Chi-squared tests or Fisher's exact test to compare qualitative variables, and t-tests or Mann-Whitney $\mathrm{U}$ and non-parametric Kruskal-Wallis tests to compare normally distributed and skewed continuous variables, as appropriate. All p-values were two-tailed. Differences were considered significant if $\mathrm{p}<0.05$.

The cumulative incidence of VA-LRTI was estimated using extubation and death as competing risks, based on the approach of Prentice et al. [22]. The cumulative incidence of VA-LRTI was compared between immunocompromised and non-immunocompromised patients using the Fine-Gray model [23]. Sub-hazard ratios (SHRs) were derived from these models, because effect size and proportional sub-hazards assumptions were assessed by examining the Schoenfeld residuals.

Univariate and multivariate analyses were used to determine factors associated with ICU mortality in patients with VA-LRTI. All variables with a p-value $<0.1$ by univariate analysis were included in a Cox proportional hazards regression model using a stepwise backwards elimination and based on a binary outcome of being discharged from the ICU dead or alive. For each candidate factor, proportional hazards were assessed by examining the Schoenfeld residuals. Effect sizes were expressed as hazard ratios.

\section{Results}

\section{Patient characteristics}

Among the 2960 included patients, 663 (22\%) had known immunosuppression, owing mainly to non-metastatic solid cancer or immunosuppressive drug use (table 1, figure 1). Age, Simplified Acute Physiology Score II, Sequential Organ Failure Assessment score, percentage of patients with chronic kidney disease and medical category of admission were significantly higher among immunocompromised patients than among non-immunocompromised patients. The percentage of patients with a history of alcohol abuse was significantly lower among immunocompromised patients than among non-immunocompromised patients. No significant difference was found between the two groups for other patient characteristics (table 2). Reasons for ICU admission are summarised in the supplementary material. 
TABLE 1 Types of immunosuppression

n (\%)

Non-metastatic solid cancer

Immunosuppressive drug

Haematological malignancy

$123(19)$

Metastatic solid cancer

$112(17)$

HIV

49 (7)

Organ transplant

$28(4)$

Allogeneic HSCT

$25(4)$

Total $\mathbf{n}$

$663^{\#}$

HSCT: haematopoietic stem cell transplant. ${ }^{\#}$ : total percentage is higher than $100 \%$ because several patients had more than one type of immunosuppression.

For patients with VA-LRTI, the rate of prior antibiotic treatment was significantly higher among immunocompromised patients than among non-immunocompromised patients (87 of 111 (78\%) versus 355 of 540 (66\%); OR 1.89, 95\% CI 1.16-3.1; p=0.009). Similar results were found in the subgroups of patients with VAT (41 of $52(79 \%)$ versus 169 of $263(64 \%)$; $=0.041)$, but not in those with VAP (46 of 59 (78\%) versus 186 of $277(67 \%) ; \mathrm{p}=0.1)$.

\section{Incidence of VA-LRTI}

The incidence of VA-LRTI was significantly lower among immunocompromised patients than among non-immunocompromised patients. Similarly, the incidence of VAT or VAP was significantly lower among immunocompromised patients than among non-immunocompromised patients (table 3). The rate of patients with early-onset VA-LRTI [19] was not significantly different between patients with immunosuppression and those with no immunosuppression (36 of 116 (31\%) versus 229 of 568 (40\%); $\mathrm{p}=0.33$ ). Similar results were found in the subgroups of patients with VAT (16 of 52 (31\%) versus 101 of 267 (38\%); $\mathrm{p}=0.32$ ) and with VAP (20 of 64 (31\%) versus 128 of $302(42 \%) ; \mathrm{p}=0.099)$.

\section{Multidrug-resistant bacteria}

In patients with VA-LRTI, the rate of MDR bacteria was significantly higher among immunocompromised patients than among non-immunocompromised patients (83 of 116 (72\%) versus 338 of 573 (59\%);

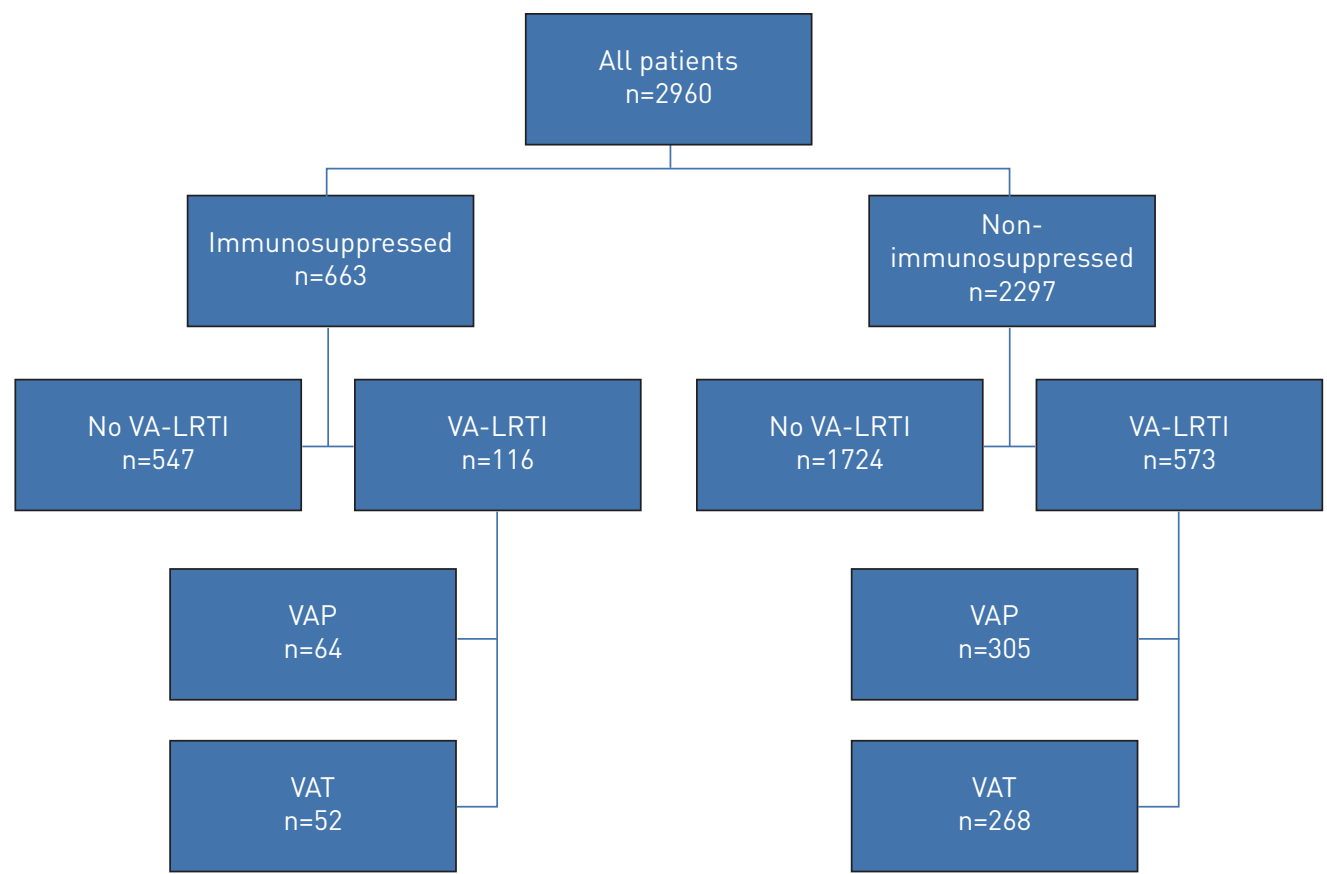

FIGURE 1 Study flowchart. VA-LRTI: ventilator-associated lower respiratory tract infections; VAP: ventilator-associated pneumonia; VAT: ventilator-associated tracheobronchitis. 
TABLE 2 Patient characteristics

\begin{tabular}{lccc} 
& \multicolumn{2}{c}{ Immunosuppression } & p-value \\
\cline { 2 - 3 } & Yes & No & \\
\hline Subjects $\mathbf{n}$ & 663 & 2297 & 0.020 \\
Age years & $63 \pm 15$ & $61 \pm 17$ & 0.51 \\
Male sex & $407(6)$ & $1442(63)$ & 0.001 \\
SAPS2 & $53 \pm 19$ & $50 \pm 19$ & 0.031 \\
SOFA & $8 \pm 4$ & $8 \pm 4$ & 0.80 \\
Chronic disease & $125(22)$ & $443(19)$ & $0.028^{\#}$ \\
$\quad$ Diabetes mellitus & $64(10)$ & $294(13)$ & 0.76 \\
Alcohol abuse & $62(9)$ & $224(10)$ & 0.78 \\
Chronic respiratory failure & $113(17)$ & $381(17)$ & $0.001^{\pi}$ \\
COPD & $88(13)$ & $205(9)$ & 0.95 \\
Chronic kidney disease & $40(6)$ & $137(6)$ & $<0.001$ \\
$\quad$ Cirrhosis & & $1435(63)$ & \\
Category of admission & $453(68)$ & $379(17)$ & \\
$\quad$ Medical & $165(25)$ & $483(21)$ & \\
$\quad$ Surgical & $45(7)$ & & \\
$\quad$ Trauma & &
\end{tabular}

Data are presented as $\mathrm{n}(\%)$ or mean \pm SD, unless otherwise stated. SAPS2: Simplified Acute Physiology Score II; SOFA: Sequential Organ Failure Assessment score; COPD: chronic obstructive pulmonary disease. "\# : OR 0.72, 95\% Cl 0.55-0.97; ๆ: OR 1.56, 95\% Cl 1.20-2.04.

OR $1.75,95 \%$ CI $1.13-2.71 ; \mathrm{p}=0.011)$. Similar results were found in the subgroup of patients with VAP (49 of 64 (78\%) versus 176 of 305 (58\%); OR 2.39, 95\% CI 1.29-4.48; $\mathrm{p}=0.005)$, but not in VAT patients (34 of $52(65 \%)$ versus 162 of 268 (60\%); $\mathrm{p}=0.52)$.

Among patients with VA-LRTI, methicillin-resistant Staphylococcus aureus and Enterobacter spp. were significantly more frequent in immunocompromised patients than in non-immunocompromised patients; however, methicillin-sensitive Staphylococcus aureus was significantly less frequent in immunocompromised patients than in non-immunocompromised patients. No significant difference was found in the incidence of other bacteria between immunocompromised and non-immunocompromised patients.

Progression from VAT to VAP

The incidence of progression from VAT to VAP was not significantly different between immunocompromised and non-immunocompromised patients (7 of 52 (13\%) versus 32 of 268 (12\%); $\mathrm{p}=0.69)$. The percentage of patients who received appropriate antibiotic treatment for VA-LRTI was not significantly different between immunocompromised and non-immunocompromised patients (77\% versus $79 \% ; \mathrm{p}=0.5$ ). Similar results were obtained in VAT and VAP subgroups (data not shown). Among immunocompromised patients with VAT, 39 of 52 (75\%) received appropriate antibiotics. The percentage of immunocompromised patients with progression from VAT to VAP was significantly lower in patients

\begin{tabular}{|c|c|c|c|c|}
\hline & \multicolumn{4}{|c|}{ Immunosuppression } \\
\hline & Yes & No & $p$-value & SHR $(95 \%$ CI) \\
\hline Subjects $n$ & 663 & 2297 & & \\
\hline VA-LRTI & $116(16.6)$ & 573 (24.2) & $<0.0001$ & $0.65(0.53-0.80)$ \\
\hline VAT & 52 (7.3) & $268(11.6)$ & 0.002 & $0.61(0.45-0.84)$ \\
\hline VAP & 64 (9.3) & 305 (12.7) & 0.019 & $0.72(0.54-0.95)$ \\
\hline
\end{tabular}

Data are presented as number of events (cumulative incidence rate in \%, calculated using competing approach), unless otherwise stated. SHRs were calculated based on Fine-Gray model, using extubation and death as competing events. SHR: sub-hazard ratio; VA-LRTI: ventilator-associated lower respiratory tract infection; VAT: ventilator-associated tracheobronchitis; VAP: ventilator-associated pneumonia. 


\begin{tabular}{|c|c|c|c|c|c|c|c|c|}
\hline & \multicolumn{8}{|c|}{ Immunosuppression } \\
\hline & \multicolumn{4}{|c|}{ Yes ${ }^{\#}$} & \multicolumn{4}{|c|}{ No" } \\
\hline & VAT & VAP & No VA-LRTI & p-value & VAT & VAP & No VA-LRTI & p-value \\
\hline Subjects n & 52 & 64 & 547 & & 268 & 305 & 1724 & \\
\hline MV duration days & $16(10-25.5)$ & 15 (8-27) & $7(4-14)$ & $<0.0001$ & $13(8-22)$ & $14(8-26)$ & $7(4-12)$ & $<0.0001$ \\
\hline ICU length of stay days & $23(16-38)$ & $20(13-30)$ & $12(7-20)$ & $<0.0001$ & $21(14-33)$ & $21(13-34)$ & $12(8-19)$ & $<0.0001$ \\
\hline
\end{tabular}

Data are presented as median (interquartile range), unless otherwise stated. p-values are for comparisons between the three groups. VAT: ventilator-associated tracheobronchitis; VAP: ventilator-associated pneumonia; VA-LRTI: ventilator-associated lower respiratory tract infection; MV: mechanical ventilation; ICU: intensive care unit. ${ }^{\#}: \mathrm{n}=663 ;{ }^{\uparrow}: \mathrm{n}=547 ;{ }^{+}$: $\mathrm{OR} 3.4,95 \% \mathrm{Cl} 1.93-5.96$ versus VAP in patients with no immunosuppression; $p<0.001$.

who received appropriate antibiotic treatment than in those who received inappropriate antibiotic treatment ( 3 of 39 (8\%) versus 4 of 13 (31\%); OR 0.19, 95\% CI $0.035-0.992 ; \mathrm{p}=0.035$ ).

Impact of immunosuppression on VA-LRTI outcomes

Among patients with VA-LRTI, ICU mortality rate was higher among immunocompromised patients than among non-immunocompromised patients (54\% versus 30\%; OR 2.68, 95\% CI 1.78-4.02; p<0.0001). Mortality was significantly higher in case of VAP in both groups, as compared to patients with no VA-LRTI and patients with VAT (table 4). In VAP patients, ICU mortality was significantly higher in immunocompromised compared with non-immunocompromised patients. Furthermore, in both groups, VA-LRTI was associated with longer duration of mechanical ventilation and ICU length of stay (table 4).

Immunosuppression was independently associated with ICU mortality in patients with VA-LRTI (table 5).

Additional results are provided in the supplementary material.

\section{Discussion}

Our results show that the incidence of VA-LRTI was significantly lower among immunocompromised patients than among non-immunocompromised patients. Additionally, immunocompromised patients more frequently received previous antibiotic treatment and developed VAP related to MDR bacteria than did non-immunocompromised patients. The development of VAT or VAP in immunocompromised patients was associated with increased duration of mechanical ventilation, and VAP was associated with significantly increased ICU mortality, as compared with non-immunocompromised patients.

To our knowledge, this is the first study on VA-LRTI in this population. The TAVeM study is the largest prospective multicentre international observational study of the natural history and incidence of VA-LRTI, and it generated robust and reproducible results [2].

The lower incidence of VA-LRTI, including VAT and VAP, in immunocompromised patients is rather surprising, as we expected that immunocompromised patients would develop more VA-LRTI than non-immunocompromised patients, given the context of immunosuppression. One could argue that we under-diagnosed these infections because of neutropenia, but only nine patients had neutropenia in our

TABLE 5 Risk factors for intensive care unit mortality in patients with ventilator-associated lower respiratory tract infection using Cox proportional hazards regression analysis

\begin{tabular}{lcc} 
Multivariate analysis & p-value & Hazard ratio (95\% CI) \\
\hline Age & 0.005 & $1.01(1.003-1.01)^{\#}$ \\
Immunosuppression & 0.002 & $1.6(1.19-2.16)^{\sharp}$ \\
SOFA score at VA-LRTI diagnosis & $<0.001$ & $1.04(1.03-1.06)^{\pi}$ \\
Appropriate antibiotic treatment & 0.005 & $0.61(0.44-0.86)$ \\
\hline
\end{tabular}

SOFA: sequential organ failure assessment; VA-LRTI: ventilator-associated lower respiratory tract infection. ${ }^{\#}$ : per year; ${ }^{\text {": }}$ per point. 
large cohort. Further, diagnostic criteria used in our study were strict, and detailed microbiology was required.

The lower incidence of VA-LRTI is probably linked to the higher exposure to previous antibiotic treatment in immunocompromised patients and, as a consequence, the higher rate of MDR-related VA-LRTI in this population. Previous randomised controlled and observational studies suggested that antibiotic treatment in mechanically ventilated patients with coma was associated with a significantly reduced incidence of early-onset VAP [24-26]. However, antibiotic treatment is a well-known risk factor for late-onset VAP related to MDR [27-29]. Previous studies clearly show that VAP related to MDR is associated with higher mortality rates than VAP related to sensitive bacteria [30-32]. Several explanations have been suggested to explain the link between MDR and mortality, including higher rates of inappropriate antibiotic treatment, patient's underlying conditions, altered antimicrobial pharmacokinetic and high minimum inhibitory concentrations, toxicity of last-resort antibiotics and emergence of subsequent resistance [33].

Even though the incidence of VAT was lower among immunocompromised patients, the rate of progression from VAT to VAP was similar between immunocompromised and non-immunocompromised patients, suggesting that immunosuppression is not a risk factor for progression from VAT to VAP. The rate of progression from VAT to VAP was low in this study, probably because most of the patients with VAT received antibiotic treatment (92\%). Interestingly, as in non-immunocompromised patients, the use of appropriate antibiotic treatment for VAT reduced the risk of progression towards VAP in immunocompromised patients. Appropriate antibiotic treatment was shown to be a protective factor in multivariate analysis for mortality risk in the TAVeM study [2]. Therefore, the early use of appropriate antibiotic treatment for tracheobronchitis in immunocompromised patients could be beneficial to reduce the transition from VAT to VAP and improve patient outcomes [34]. However, few studies have focused on antibiotic treatment for VAT $[35,36]$, and further large multicentre randomised controlled studies are required to clarify this issue [4].

In spite of similar rates of appropriate initial antibiotic treatment, immunocompromised patients with VA-LRTI had higher mortality rates than non-immunocompromised patients, mainly due to higher mortality among immunocompromised patients with VAP. Further, immunosuppression was independently associated with ICU mortality among patients with VA-LRTI. Previous studies reported higher associated-mortality rates among VAP patients [8, 37-39]. However, the mortality attributable to VAP is still a matter for debate, and is probably low [10]. In addition, the higher mortality among immunocompromised patients admitted to the ICU is already well described and is not surprising [17]. To our knowledge, no study has evaluated the relationship between mortality and immunosuppression in VAP patients.

Taken together, our results suggest that antibiotic treatment should be reduced and better tailored in immunocompromised patients to prevent VAP related to MDR in this population. Further, preventive

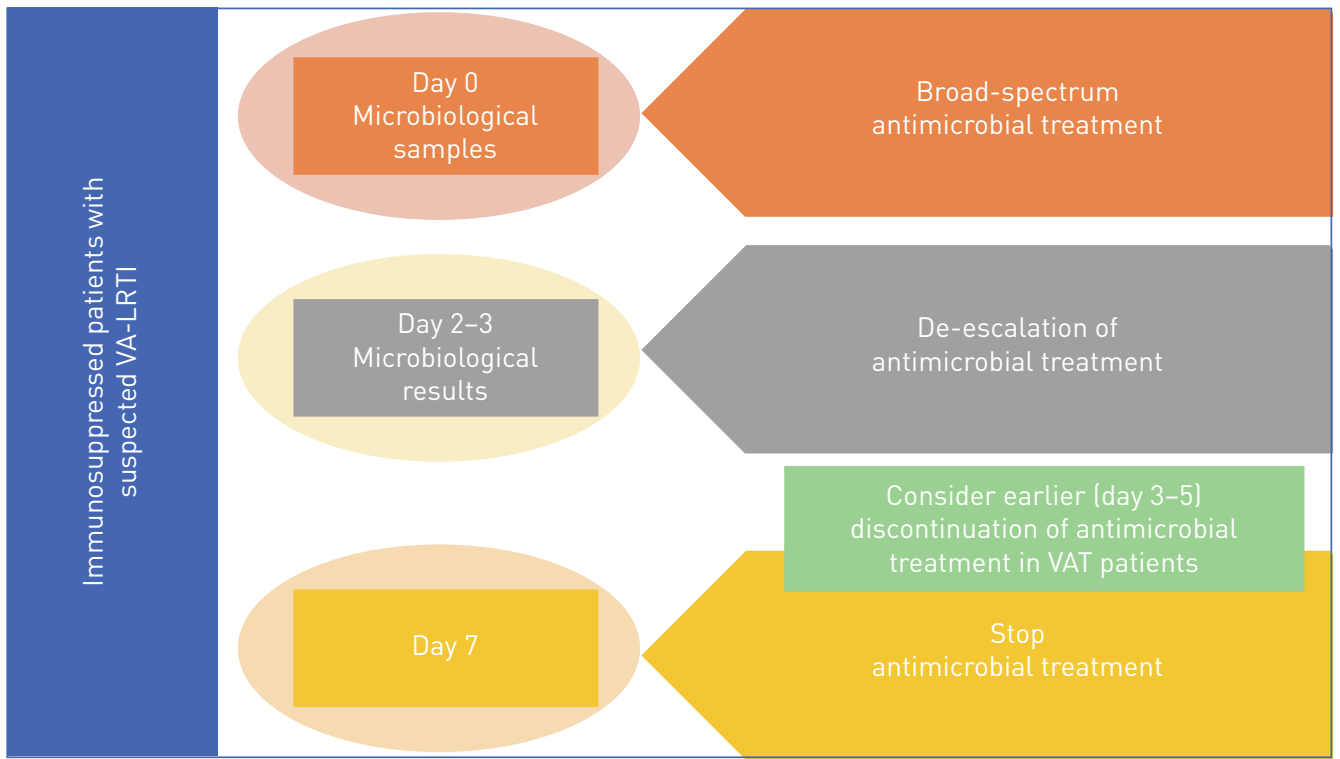

FIGURE 2 Suggested algorithm for antibiotic treatment in immunosuppressed patients with ventilator-associated lower respiratory tract infections (VA-LRTI). VAT: ventilator-associated tracheobronchitis. 
measures should be enhanced in immunocompromised patients, and appropriate initial antibiotic treatment should be the gold standard in these patients. Our data also suggest that broad-spectrum antibiotic treatment should be given to immunocompromised patients with VA-LRTI, and that de-escalation should be performed as soon as possible after receipt of microbiological results to break the vicious circle of overuse of antimicrobials and MDR emergence [40, 41]. However, further data are required on the relationship between de-escalation and outcome in immunocompromised patients [42]. The use of procalcitonin could be helpful to encourage physicians to perform de-escalation. Given the lower severity of VAT, as compared with VAP, and the absence of impact on mortality, one could argue that VAT could be treated by a shorter duration of antimicrobial treatment even in immunocompromised patients (figure 2).

Our study has some limitations. Our immunocompromised group included patients with many types of immunosuppression, and the number of patients with neutropenia was small. However, this is the first study on this particular population and we believe that our results are robust, and might be helpful for future research.

\section{Conclusions}

The incidence of VA-LRTI is significantly lower among immunocompromised patients than among non-immunocompromised patients. These infections are more frequently caused by MDR in this population, and are associated with substantially higher mortality. These results suggest that prior antibiotic treatment should be better tailored in immunocompromised patients to reduce the incidence of MDR-related infections. Further studies are required to better determine the relationship between the type of immunosuppression and the risk for VA-LRTI.

The TAVeM Study Group included the following contributors: Ignacio Martín-Loeches, Alejandro Rodriguez, Daniel Curcio, Rubén Oscar Fernández, Jorge Arroyo, Maria Gabriela, Rodriguez Alvarez, Alex Tamayo Reyes, Christian Dellera, Francisco Molina, Daniel Molano Franco, Edwin Giovanny Chapeta Parada, Estuardo Salgado Yepez, Fernando Paredes Oña, Diego Morocho Tutillo, Diego Barahona, Francisco Alvarez Lerma, Ana Abella Álvarez, Jose Manuel Allegue Gallego, Francisco José Fuentes Morillas, Antonio Luis Ruiz Aguilar, María Lourdes Cordero Lorenzana, Rafael Sanchez Iniesta, Jordi Almirall, Antonio Albaya, Sergio Ruiz Santana, Carmen Fernandez, Miguel Angel Blasco Naval Potro, Pablo Vidal Cortes, Belen Jimenez, Rafael Sierra, Maria Del Valle Ortiz, Nieves Cruza, Pedro Maria Olaechea, Ana Carolina Caballero Zirena, Pilar Posada Gonzalez, Teresa Recio Gomez, Lorenzo Socias Crespi, Paula Ramírez Galleymore, Ricard Jordà Marcos, César Palazón, Bernardo Gil Rueda, Juan Carlos Ballesteros, Maria Pilar Gracia Arnilla, Antonia Socias, Joaquin Amador, Esperanza Molero Silvero, Laura Macaya Redín, Mónica Zamora Elson, Luis Cabré Pericas, Joaquín Álvarez Rodríguez, Mercedes Nieto, Antoni Torres, Elena Molinos, Ana Josefina, Nuno Catorze, Pedro Póvoa, Carlos Candeias, Luis Coelho, Paulo André, José Andrade Gomes, Elisa Vedes, Antero Fernandes, Miguel Ángel García, Catalina Sanchez Ramirez, Milagros Calizaya, Angel Estella, Adrià Albis, Gerardo Aguilar, Eva Torrents, Marta Gurpegui Puente, Angel Gabriel Sanchez, Thiago Lisboa, Pedro Azambuja, Marcos Freitas Knibel, Otavio Ranzani, Laura Darriba W. Camargo, Antonio Paulo Nassar Junior, Cesar Biselli Ferreira, Suzana Lobo, Ligia Rabello, Marcelo Park, Alexandre Guilherme Ribeiro de Carvalho, Mauricio Valencia, Alejandro Gonzalez Castro, Adoración Alcalá López, José María Castillo Caballero, Saad Nseir, Karim Jaffal, Erika Parmentier-Decrucq, Sébastien Préau, Chloé Rousselin, Caroline Blazejewski, Juliette Masse, Laurent Robriquet, Léa Satre-Buisson, Jean-Paul Mira, Nathalie Martin, Raphael Lepecq, Hervé Mentec, Christophe Girault, Antoine Marchalot, Jonathan Messika, Jean-Damien Ricard, Philippe Seguin, Bruno Mégarbane, Sandrine Valade, Elie Azoulay, Nicolas Boussekey, Olivier Leroy, Jean Reignier, Marc Clavel, Nicolas Pichon, Thomas Baudry, Laurent Argaud, Pascal Beuret, Ali Ait-Hssain, Martine Nyunga, Isabelle Alves, Florent Dewavrin, Guillaume Brunin, Stéphane Mérat, Pierre Pasquier, Frédéric Brun, Aurore Palud, Benoit Voisin, Romaric Grenot, Nicolas Van Grunderbeeck, Didier Thévenin, Benoit Misset, François Philippart, Jean-Pierre Frat, Rémi Coudroy, Philippe Cabaret, Marie Ledein, Fethi Zoheir Hadj Slimane, Romain Miguel-Montanes, Nicolas Weiss, Francis Bolgert, Bernard Just.

Conflict of interest: S. Nseir has received personal fees (for advisory board work) from Bayer and Ciel Medical, and personal fees (for lectures) from MSD and Medtronic, outside the submitted work.

\section{References}

1 Craven DE, Hudcova J, Rashid J. Antibiotic therapy for ventilator-associated tracheobronchitis: a standard of care to reduce pneumonia, morbidity and costs? Curr Opin Pulm Med 2015; 21: 250-259.

2 Martin-Loeches I, Povoa P, Rodríguez A, et al. Incidence and prognosis of ventilator-associated tracheobronchitis (TAVeM): a multicentre, prospective, observational study. Lancet Respir Med 2015; 3: 859-868.

3 Rodriguez A, Povoa P, Nseir S, et al. Incidence and diagnosis of ventilator-associated tracheobronchitis (VAT) in the intensive care unit: an international online survey. Crit Care 2014; 18: R32.

4 Nseir S, Povoa P, Salluh J, et al. Is there a continuum between ventilator-associated tracheobronchitis and ventilator-associated pneumonia? Intensive Care Med. 2016; 42: 1190-1192.

5 Nseir S, Di Pompeo C, Pronnier P, et al. Nosocomial tracheobronchitis in mechanically ventilated patients: incidence, aetiology and outcome. Eur Respir J 2002; 20: 1483-1489.

6 Karvouniaris M, Makris D, Manoulakas E, et al. Ventilator-associated tracheobronchitis increases the length of intensive care unit stay. Infect Control Hosp Epidemiol 2013; 34: 800-808.

7 Craven DE, Lei Y, Ruthazer R, et al. Incidence and outcomes of ventilator-associated tracheobronchitis and pneumonia. Am J Med 2013; 126: 542-549. 
8 Melsen WG, Rovers MM, Groenwold RHH, et al. Attributable mortality of ventilator-associated pneumonia: a meta-analysis of individual patient data from randomised prevention studies. Lancet Infect Dis 2013; 13: 665-671.

9 Parmentier-Decrucq E, Nseir S, Makris D, et al. Accuracy of leptin serum level in diagnosing ventilator-associated pneumonia: a case-control study. Minerva Anestesiol 2014; 80: 39-47.

10 Bekaert M, Timsit JF, Vansteelandt S, et al. Attributable mortality of ventilator-associated pneumonia: a reappraisal using causal analysis. Am J Respir Crit Care Med 2011; 184: 1133-1139.

11 Branch-Elliman W, Wright SB, Howell MD. Determining the ideal strategy for ventilator-associated pneumonia prevention. Cost-benefit analysis. Am J Respir Crit Care Med 2015; 192: 57-63.

12 Melsen WG, Rovers MM, Bonten MJM. Ventilator-associated pneumonia and mortality: a systematic review of observational studies. Crit Care Med 2009; 37: 2709-2718.

13 Darmon M, Ranzani OT, Azoulay E. Focus on immunocompromised patients. Intensive Care Med 2017; 43: 1415-1417.

14 Soares M, Bozza FA, Azevedo LCP, et al. Effects of organizational characteristics on outcomes and resource use in patients with cancer admitted to intensive care units. J Clin Oncol 2016; 34: 3315-3324.

15 Nseir S, Di Pompeo C, Diarra M, et al. Relationship between immunosuppression and intensive care unit-acquired multidrug-resistant bacteria: a case-control study. Crit Care Med 2007; 35: 1318-1323.

16 Azoulay E, Lemiale V, Mokart D, et al. Acute respiratory distress syndrome in patients with malignancies. Intensive Care Med 2014; 40: 1106-1114.

17 Azoulay E, Mokart D, Pène F, et al. Outcomes of critically ill patients with hematologic malignancies: prospective multicenter data from France and Belgium--a Groupe de Recherche Respiratoire en Réanimation Onco-Hématologique study. J Clin Oncol 2013; 31: 2810-2818.

18 Lemiale V, Resche-Rigon M, Azoulay E, Study Group for Respiratory Intensive Care in Malignancies [Groupe de Recherche en Réanimation Respiratoire du patient d'Onco-Hématologie, GRRR-OH]. Early non-invasive ventilation for acute respiratory failure in immunocompromised patients (IVNIctus): study protocol for a multicenter randomized controlled trial. Trials 2014; 15: 372 .

19 American Thoracic Society; Infectious Diseases Society of America. Guidelines for the management of adults with hospital-acquired, ventilator-associated, and healthcare-associated pneumonia. Am J Respir Crit Care Med 2005; 171: 388-416.

20 Paul M, Shani V, Muchtar E, et al. Systematic review and meta-analysis of the efficacy of appropriate empiric antibiotic therapy for sepsis. Antimicrob Agents Chemother 2010; 54: 4851-4863.

21 Magiorakos A-P, Srinivasan A, Carey RB, et al. Multidrug-resistant, extensively drug-resistant and pandrug-resistant bacteria: an international expert proposal for interim standard definitions for acquired resistance. Clin Microbiol Infect 2012; 18: 268-281.

22 Prentice RL, Kalbfleisch JD, Peterson AV, et al. The analysis of failure times in the presence of competing risks. Biometrics 1978; 34: 541-554.

23 Fine JP, Gray GR. A proportional hazards model for the subdistribution of a competing risk. J Am Stat Assoc 1999; 94: 496-509.

24 Acquarolo A, Urli T, Perone G, et al. Antibiotic prophylaxis of early onset pneumonia in critically ill comatose patients. A randomized study. Intensive Care Med 2005; 31: 510-516.

25 Sirvent JM, Torres A, El-Ebiary M, et al. Protective effect of intravenously administered cefuroxime against nosocomial pneumonia in patients with structural coma. Am J Respir Crit Care Med 1997; 155: 1729-1734.

26 Vallés J, Peredo R, Burguenõ MJ, et al. Efficacy of single-dose antibiotic against early-onset pneumonia in comatose patients who are ventilated. Chest 2013; 143: 1219-1225

27 Bassetti M, De Waele JJ, Eggimann P, et al. Preventive and therapeutic strategies in critically ill patients with highly resistant bacteria. Intensive Care Med 2015; 41: 776-795.

28 Martín-Loeches I, Diaz E, Vallés J. Risks for multidrug-resistant pathogens in the ICU. Curr Opin Crit Care 2014; 20: 516-524

29 Garnacho-Montero J, Dimopoulos G, Poulakou G, et al. Task force on management and prevention of Acinetobacter baumannii infections in the ICU. Intensive Care Med 2015; 41: 2057-2075.

30 Depuydt PO, Vandijck DM, Bekaert MA, et al. Determinants and impact of multidrug antibiotic resistance in pathogens causing ventilator-associated-pneumonia. Crit Care 2008; 12: R142.

31 Martin-Loeches I, Torres A, Rinaudo M, et al. Resistance patterns and outcomes in intensive care unit (ICU)-acquired pneumonia. Validation of European Centre for Disease Prevention and Control (ECDC) and the Centers for Disease Control and Prevention (CDC) classification of multidrug resistant organisms. J Infect 2015; 70: 213-222.

32 Guillamet CV, Kollef MH. Update on ventilator-associated pneumonia. Curr Opin Crit Care 2015; 21: 430-438.

33 Barbier F, Lisboa T, Nseir S. Understanding why resistant bacteria are associated with higher mortality in ICU patients. Intensive Care Med 2015; 42: 2066-2069.

34 Nseir S, Martin-Loeches I, Makris D, et al. Impact of appropriate antimicrobial treatment on transition from ventilator-associated tracheobronchitis to ventilator-associated pneumonia. Crit Care 2014; 18: R129.

35 Palmer LB, Smaldone GC, Chen JJ, et al. Aerosolized antibiotics and ventilator-associated tracheobronchitis in the intensive care unit. Crit Care Med 2008; 36: 2008-2013.

36 Nseir S, Favory R, Jozefowicz E, et al. Antimicrobial treatment for ventilator-associated tracheobronchitis: a randomized, controlled, multicenter study. Crit Care 2008; 12: R62.

37 Kollef MH. Ventilator-associated pneumonia. A multivariate analysis. JAMA 1993; 270: 1965-1970.

38 Messika J, Magdoud F, Clermont O, et al. Pathophysiology of Escherichia coli ventilator-associated pneumonia: implication of highly virulent extraintestinal pathogenic strains. Intensive Care Med 2012; 38: 2007-2016.

39 Nair GB, Niederman MS. Ventilator-associated pneumonia: present understanding and ongoing debates. Intensive Care Med 2014; 41: 34-48.

40 Garnacho-Montero J, Gutiérrez-Pizarraya A, Escoresca-Ortega A, et al. De-escalation of empirical therapy is associated with lower mortality in patients with severe sepsis and septic shock. Intensive Care Med 2014; 40: 32-40.

41 Leone M, Bechis C, Baumstarck K, et al. De-escalation versus continuation of empirical antimicrobial treatment in severe sepsis: a multicenter non-blinded randomized noninferiority trial. Intensive Care Med 2014; 40: 1399-1408.

42 Mokart D, Slehofer G, Lambert J, et al. De-escalation of antimicrobial treatment in neutropenic patients with severe sepsis: results from an observational study. Intensive Care Med 2014; 40: 41-49. 